\title{
A Computational Comparison of the Multiple-breath Washout and Forced Oscillation Technique as Markers of Bronchoconstriction
}

\author{
Brody H. Foy*, David Kay \\ Department of Computer Science, University of Oxford, Oxford, Oxfordshire, United \\ Kingdom
}

*Corresponding author
Email address: brody.foy@new.ox.ac.uk (Brody H. Foy* ) 


\begin{abstract}
Many types of lung disease lead to constriction of airways within the conducting zone, causing increases in airway resistance and ventilation heterogeneity $(\mathrm{VH})$. The multiple-breath washout (MBW) and forced oscillation technique (FOT) are two clinical measures of small airway bronchoconstriction. Within this study computational modelling of the MBW and FOT was applied to the analysis of thousands of bronchoconstriction scenarios in the conducting zone of the lungs. The simulations show that MBW indices primarily respond to the severity of constriction, while FOT indices may be able to distinguish between constrictions at differing airway depths. Following this, we show the potential utility in the joint interpretation of indices from the two tests, providing motivations for future clinical investigations.
\end{abstract}

Keywords: Inert-gas washout, Forced oscillation technique, ventilation heterogeneity, airway resistance, bronchoconstriction. 


\section{Introduction}

A characteristic feature of asthma is bronchoconstriction, particularly in the small conducting airways. Typically measurements of airway constriction are made through simple lung function tests such as spirometry and plethysmog-

5 raphy. However, recently more complex lung function tests have experienced increased clinical uptake, due to potential improvements in sensitivity.

One such test is the multiple-breath washout (MBW) (Robinson et al., 2013). During the MBW test gas exhalation is monitored over two distinct phases, a wash-in and a washout. During wash-in, air mixed with a fixed percentage of have equilibrated in the lungs. Following this, the patient is switched to an air mixture without the tracer gas, and exhalation of the gas is monitored. When performing an $N_{2}$ washout, no wash-in is performed, due to the presence of $N_{2}$ in air; instead, a washout is performed using pure oxygen. A series of different Clearance Index (LCI), $s_{\text {cond }}$ and $s_{\text {acin }}$ (Verbanck et al., 1998) (for exact definitions of each, the reader is referred to Appendix A). Modelling studies (Tawhai and Hunter, 2001, Verbanck and Paiva, 1990) have helped improve understanding of how the test indices respond to morphological changes in lung structure. have highlighted the response of MBW indices to bronchoconstriction severity, but not to depth. Overall though, there is still uncertainty of whether these responses can be utilised in a clinical setting.

Alongside the MBW, the forced oscillation technique (FOT) is another complex lung function test (Smith et al. 2005). In short, this technique is performed by applying oscillations of varying frequencies at the mouth. Measurements of central airflow and pressure are made using a pneumotachograph, and used to estimate resistance $(R)$, and reactance $(X)$ of the lungs; the in and out of phase relationships between pressure and airflow, known collectively as impedance.

Typically, impedance exhibits frequency dependence, the nature of which 
fan be strongly affected by bronchoconstriction Grimby et al., 1968, Lutchen and Gillis, 1997; Oostveen et al., 2003). Due to this dependency, many different impedance derived indices are taken as markers of airway constriction. The accumulation of both clinical and morphometric modelling studies has led to the interpretation of low frequency resistance as a measure of total airway constriction, high frequency resistance as a measure of central airway constriction (Goldman et al. 2005, Smith et al., 2005) and reactance indices as sensitive to distal airway constriction (Cavalcanti et al., 2006, Kaczka et al., 1999, 2007). While it is clear that FOT indices respond to bronchoconstriction, the precise way this response occurs in relation to factors such as constriction depth and severity is not yet fully understood.

Within this study, we apply computational modelling to investigate how MBW and FOT indices respond to different types of bronchoconstriction in the conducting zone of the lungs. Given that MBW indices have been shown

45 to be sensitive to constriction severity and certain FOT indices to depth, we investigate the joint interpretation of indices derived from the two techniques. We hypothesise the potential utility of combined interpretation of the indices in separation of different types of bronchoconstriction.

\section{Methods}

\subsection{Airway Structure}

To simulate the two techniques, a structural representation of the conducting zone of the lungs was taken from a healthy subject in the set originally presented by Bordas et al. (2015). This structure was created through a combination of reconstruction of multi-slice CT scans (for generations 6-10), and algorithmic generation to an average generation 16 (Fetita et al. 2004, Tawhai et al., 2004). The airway tree structure consisted of 78,834 branches, 39,420 of which were terminal bronchioles.

To ensure no bias from the base structure all airway diameter data was discarded, and regenerated using Horsfield's idealised airway radius model (Hors- 
${ }_{60}$ field et al. 1976). Further details of the airway tree are given in Appendix B.

\subsection{Model of the $M B W$}

To model the MBW, we use the scheme previously outlined by Foy et al. (Foy et al., 2017), based in part on previous models in the literature (Mitchell

65 et al. 2012; Tawhai and Hunter, 2001; Verbanck and Paiva, 1990). In short, each terminal bronchiole is connected to an acinar volume, whose expansion and contraction is driven by flow rates, the pressure gradient between the pleural cavity and trachea, and regional tissue compliance. Flow rates are driven by the pressure gradient over each branch, with conservation at each bifurcation,

70 and Poiseuille resistance with Pedley's correction term for energy dissipation (Pedley et al. , 1970). The pleural pressure was taken as constant in space, and sinusoidal in time, with a breathing period of 4 seconds. The amplitude was chosen to enforce tidal breathing of 1 litre per cycle, above a functional residual capacity of $2.5 \mathrm{~L}$.

75 Total lung compliance was set as $0.2 \mathrm{~L} \mathrm{cmH}_{2} \mathrm{O}^{-1}$ (Galetke et al., 2007, Mittman et al., 1965), and distributed over the lungs using a linear gravitational gradient, in the cranio-caudal axis, with strength $1.5 \% \mathrm{~cm}^{-1}$ (Hopkins et al. 2007, Kaneko et al., 1966, Musch et al., 2002). A linear distribution was chosen over more complex models (Swan et al., 2012) due to computational constraints.

Information from the ventilation model was used to drive a convection transport model for gas concentration:

$$
\frac{\partial A C}{\partial t}=-u \frac{\partial A C}{\partial x},
$$

where $x$ is the axial direction of the flow, $t$ is time, $C(x, t)$ is the concentration of the gas within air, $A$ is the cross-sectional area of the branch, and $u$ is the air velocity. The radius of each branch was taken as static over the breathing cycle, with differences in cross-sectional area between connected branches accounted for in the finite volume formulation of the model. 
The branch network was started with an initial gas concentration of $0 \%$. During wash-in, inhaled gas entered the system at the trachea proportional to the tracheal flow rate, assuming an external concentration of $4 \%$. The washin was continued for long enough to assume equilibration of gas within the lungs. During the washout phase, an inspiratory inflow concentration of $0 \%$ was enforced, and during expiration gas was removed from the trachea proportional to the tracheal flow rate.

\subsection{FOT Model}

To simulate the FOT on the structural lung representation, we apply an electrical circuit analogous model (Bhatawadekar et al., 2015, Kaczka et al., 2007; Lutchen and Gillis, 1997). Each branch in the lungs is considered to have an impedance due to oscillatory flow, with each terminal airway being connected to a viscoelastic acinar unit. The total impedance of the system was then calculated through summation of series and parallel branches in the network.

In short, the oscillatory flow impedance calculations were based on the work of Benade (Benade, 1968) and Thurston (Thurston, 1952), under the assumption of a sinusoidal waveform. Each terminal bronchiole was subtended by a constant-phase viscoelastic model (Kaczka et al., 2007; Lutchen and Gillis, 1997), parameterised by clinical data (Kaczka et al., 1997). For specifics of the model, the reader is referred to Appendix C.

\subsection{Selection of bronchoconstriction scenarios}

Throughout this study, bronchoconstriction was classified according to the severity of the applied constriction, and the depth that it was applied at. Constriction severity was defined as the percentage reduction in branch radius. Strahler order (Strahler, 1957) (distance in number of branches to the nearest terminal bronchiole) was chosen as the primary marker of depth. Branches were classified into three groups: distal (Strahler orders 1-3), medial (orders 4-7) and central (orders 8-10). These groupings approximately correspond to 
the small conducting bronchioles, large conducting bronchioles and tertiary and secondary bronchi (although excluding the trachea and left and right primary bronchus).

For each simulation a depth (distal, medial, central) and mean constriction severity $\mu_{\text {cond }}(0-60 \%)$ were randomly chosen. Each branch in the chosen depth group was then constricted, with severities $(c)$ drawn from the normal distribution

$$
c \sim N\left(\mu_{\mathrm{con}}, 0.2 \mu_{\mathrm{con}}\right) .
$$

The constrictions were applied randomly to every branch in the chosen depth ground, with no preference given to branch sizes or locations. If any constriction rate exceeded $95 \%$ it was floored to this value to prevent non-physical scenarios. The value of $0.2 \mu_{\text {con }}$ was chosen as the standard deviation to provide a high degree of variability and noise to the constriction protocol. For an analysis of the effect of this choice the reader is referred to supplemental materials.

For each simulation, resistance and reactance were generated over the frequency range $2-25 \mathrm{~Hz}$. The resistance at $5\left(R_{5}\right)$ and $20 \mathrm{~Hz}\left(R_{20}\right)$ were calculated, as well as the absolute value of reactance at $5 \mathrm{~Hz}\left(\left|X_{5}\right|\right)$, the resonant frequency $\left(f_{\text {res }}\right)$ and the low reactance area $(A X)$. These parameters are outlined in Fig. 1] Under the same constriction pattern, a multiple-breath washout curve was generated, and the corresponding LCI and $s_{\text {cond }}$ values calculated. In this study, 1200 bronchoconstriction scenarios were simulated.

\subsection{Software}

All models were implemented numerically in MATLAB. Differential equations were solved using Backward Euler discretisations, coupled with vector Newton Methods, and Krylov subspace techniques. Mass conservation was verified, and spatial and temporal step sizes were chosen to ensure solution changes of less than $1 \%$ upon further reduction. 

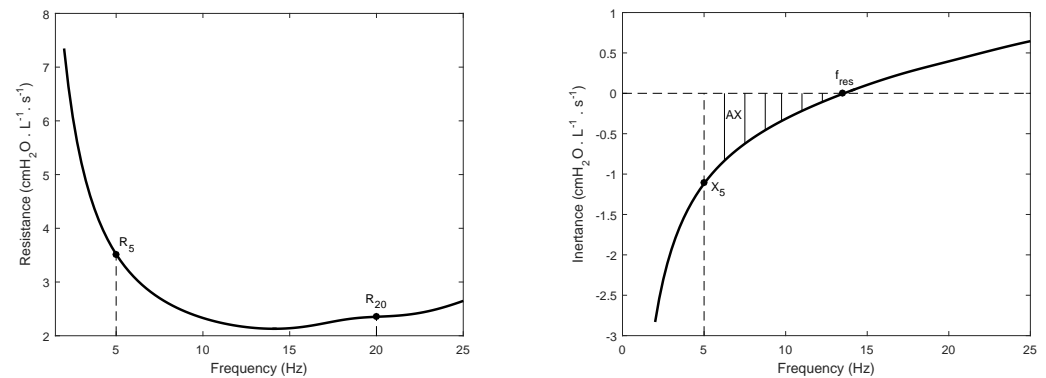

Figure 1: Examples of resistance (left) and reactance (right) curves. The key FOT parameters, resistance at $5 \mathrm{~Hz}\left(R_{5}\right)$, at $20 \mathrm{~Hz}\left(R_{20}\right)$, reactance at $5 \mathrm{~Hz}\left(X_{5}\right)$, resonant frequency $\left(f_{\text {res }}\right)$, and low reactance area $(A X)$ are all indicated.

\section{Results}

For reference, we give the baseline values of the model (produced under no constriction) as $0.3478,0.3154$, and $0.4779 \mathrm{cmH}_{2} \mathrm{O} . \mathrm{L}^{-1} \cdot s^{-1}$ for $R_{5}, R_{20}$ and $\left|X_{5}\right|$ respectively, $8.79 \mathrm{~Hz}$ for $f_{\text {res }}, 0.816 \mathrm{~Hz} . \mathrm{cmH}_{2} \mathrm{O} . \mathrm{L}^{-1} \cdot \mathrm{s}^{-1}$ for $\mathrm{AX}, 0.0012 \mathrm{~L}^{-1}$ for $s_{\text {cond }}$ and 4.948 turnovers for the Lung Clearance Index.

\subsection{Response of $M B W$ indices}

In Fig 2 we show the response of $s_{\text {cond }}$ and LCI to increases in mean constriction severity. As the figure shows, both indices exhibit a clear positive response to increasing severity. The index $s_{\text {cond }}$ appears to show some ability to distinguish between the three depth groups (in particular the distal group). However, similarly to the resistance indices, there is a high degree of variability to the responses, meaning there is significant overlap between the three groups. Considering the responses of LCI, we see the formation of distinct bands. This is due to the discrete threshold used in the calculation of LCI, meaning small changes in VH can cause step-wise changes in the index. Due to this phenomenon, LCI appears to separate depth groups less clearly than $s_{\text {cond }}$.

\subsection{Responses of FOT indices}

In Fig 3 we give the responses of the six FOT indices to increases in mean constriction severity. Results have been separated into the three depth groups: 

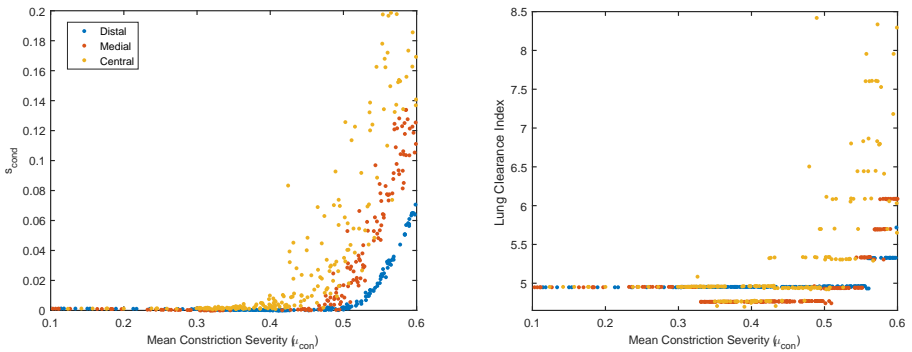

Figure 2: Response of the two MBW indices to increases in mean constriction severity. Results have been separated by the three constriction groups: distal (blue), medial (red) and central (yellow).

distal, medial and central. Considering the resistance indices, both $R_{5}$ and $R_{20}$ show clear positive responses to increasing constriction severity. However, neither $R_{5}$ nor $R_{20}$ show clear distinctions between the three depth groups. The third resistance index, $R_{5}-R_{20}$ appears to give more distinction between depths, with the distal group having a significantly lowered response (though this may in part be due to the smaller value range of the index). As expected, $R_{5}-R_{20}$ has a significantly higher variance (being the difference of two variables) than the other resistance indices.

Similarly to the resistance indices, each reactance index responds strongly to increases in constriction severity; however, the nature of this response differs between each depth group. For all three indices, the distal and medial groups experience monotonic increases, while the central group decreases, before eventually increasing as the mean constriction becomes severe. Unlike the resistance indices, the reactance indices show distinct separations between the medial and distal groups.

\subsection{Joint response of indices}

In Fig 4 we show the comparative response of $s_{\text {cond }}$ and the FOT indices. As the figure shows, for each of the subplots the three depth groups are distinctly separated. Equally, as shown by the arrows, there is clear separation based on mean constriction severity. The distinction between groups is most clear with 

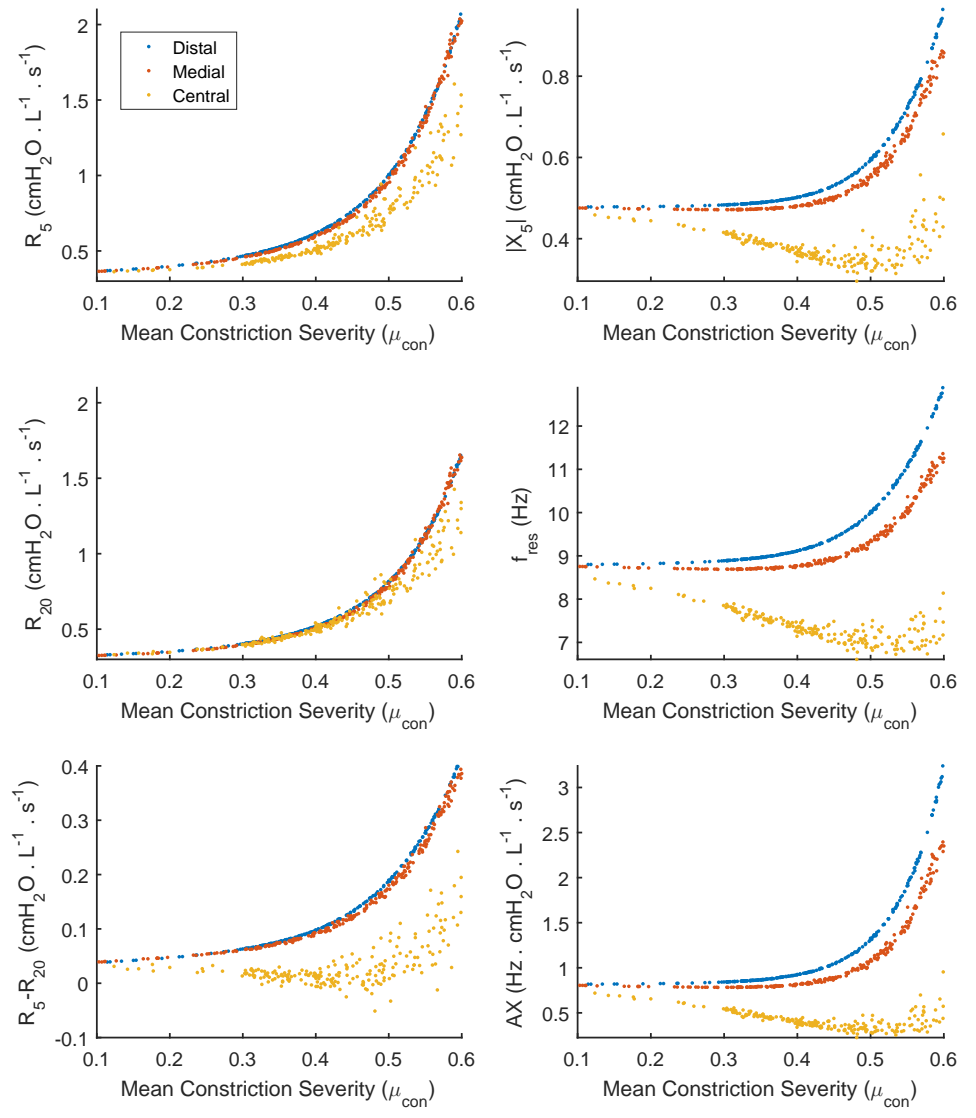

Figure 3: Response of the six FOT indices to increases in mean constriction severity. Results have been separated by the three constriction groups: distal (blue), medial (red) and central (yellow).

the reactance indices. This is logical, as the results from Fig 2 and 3 suggest that $s_{\text {cond }}$ responds strongly to constriction severity, while the reactance indices respond strongly to depth. 

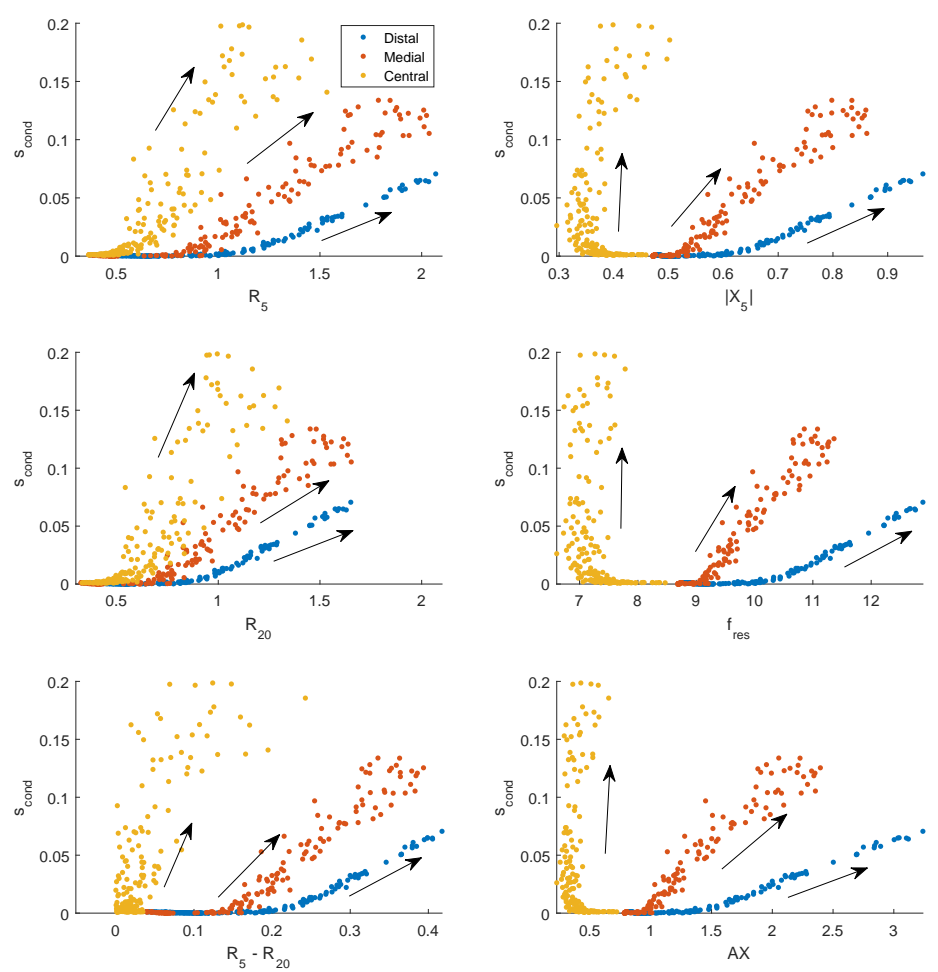

Figure 4: Comparative responses of the FOT indices and $s_{\text {cond }}$. Results have been separated by the three constriction groups: distal (blue), medial (red) and central (yellow). Directions of increasing constriction severity for each depth group are marked by arrows.

\section{Discussion}

In this study the MBW and FOT were simulated under a range of different bronchoconstriction scenarios, applied to a structural representation of the lungs derived from segmentation of CT data, and algorithmic branch generation. Within the results (Fig. 2 and 3 MBW indices were shown to respond positively to increases in mean constriction severity, with the majority of responses of $s_{\text {cond }}$ and all responses of LCI occurring within ranges seen in clinical studies (Verbanck et al. 1997, 2004). Similarly, all FOT indices responded positively 
to increases in constriction severity, with the reactance responses also occurring within ranges seen in clinical studies (Crim et al., 2011). The baseline resistances of the model were lower than standard clinical measurements, potentially owing to the non-compliant and idealised airway radii. However, under constriction the values increased into experimentally validated ranges.

The MBW showed only small responses to changes in constriction depth, while $R_{5}-R_{20}$ and the reactance indices all showed strong distinctions between depth groups, peaking distally. These trends are consistent with prior results in the literature (Foy et al., 2017; Goldman et al., 2005; Smith et al., 2005) and provide some validation to the model.

Considering the results in Fig. 2 and 3, the results in Fig. 4 are not surprising. However, to the best of our knowledge, no prior study has investigated this connection. As the figure shows, the joint interpretation of MBW and FOT indices provides separation of the simulations based on both constriction depth and constriction severity, with an example schematic representation of this given in Fig. 5 .

It is noteworthy that the MBW and FOT computational models operate completely independently of each other. The only shared information between the two tests is the branch structure, and airway constriction pattern. No parameters are shared between the two models, and the underlying model assumptions and mechanics are quite different, with the MBW model simulating ventilation and gas transfer, while the FOT model simulates frequency propagation. This makes the clear and distinct separation of groups, and the low level of variance within each group quite fascinating.

The constriction distribution used in this study was not intended to be a true representation of asthmatic bronchoconstriction, but rather to explore in a broad sense the responses of the two tests to bronchoconstriction depth and severity. Clearly experimental measurements would be needed to be certain of the physiological interpretation of the observed relationships within this study. However, these results can be taken as a hypothesis-generating explorative study, suggesting the potential ways a lung might respond to bronchoconstriction. We believe 


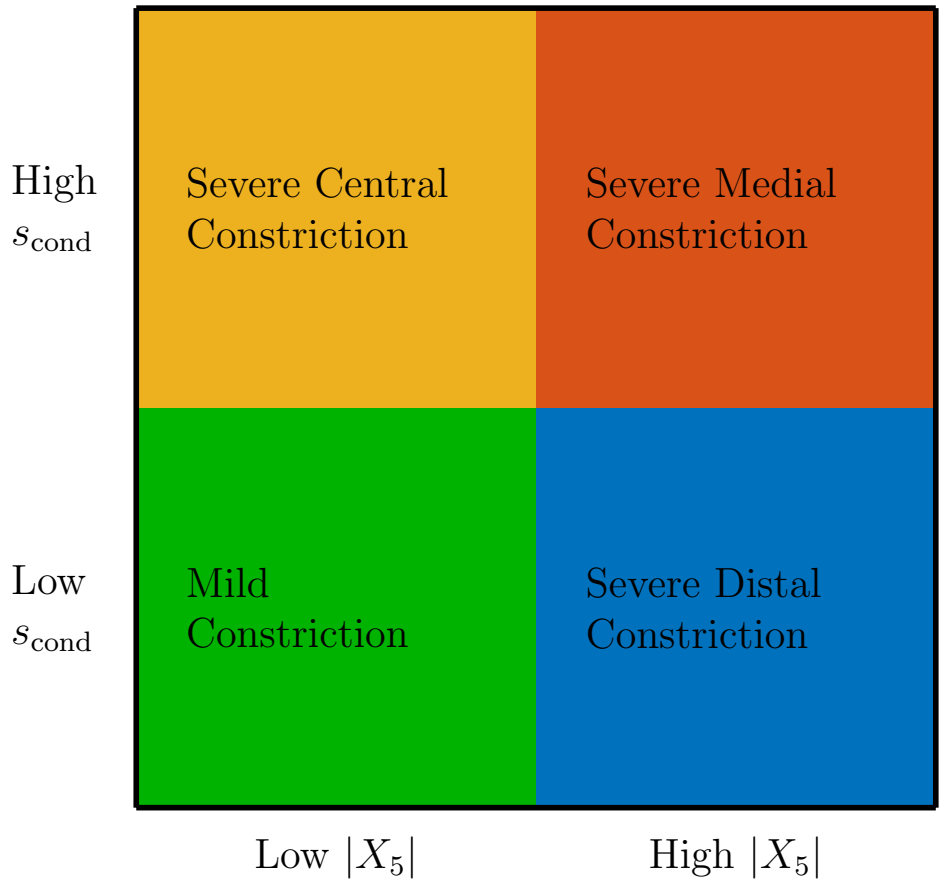

Figure 5: An illustration of the combined interpretation of the indices derived from the two computational models, separating out constriction severity and depth.

that the hypothesised relationship of the MBW and FOT to bronchoconstriction presented in Fig 5 is one that warrants further investigation in an clinical setting.

Constrictions to the airway tree were drawn from a normal distribution, with a uniformly randomly chosen mean $\mu_{\mathrm{con}}$, and standard deviation $0.2 \mu_{\mathrm{con}}$. In many computational studies of bronchoconstriction, constrictions are instead drawn uniformly (Foy et al., 2017, Mitchell et al. 2012, Lutchen and Gillis, 1997). Increases in constriction heterogeneity may create a greater spectrum of time constants (Otis et al. 1956) in the lung, and as such should lead to higher $s_{\text {cond }}$ values. Comparing results in this study to the results of Foy et al. (2017) and Mitchell et al. (2012) we see that this occurs. This is further illustrated in the supplemental material (which applies constrictions less heterogeneously), suggesting stronger heterogeneity may lead to greater index responses. Com- 
paring results within this study to prior results in the literature Lutchen and Gillis, 1997), similar behaviours can be seen in the FOT indices, with the degree of heterogeneity of the applied constriction affecting index magnitudes. Since increased heterogeneity appears to cause increases in both MBW and FOT indices, the conclusions seen in this study should remain consistent if constriction heterogeneity is changed.

Within the results, the MBW indices exhibited less sensitivity to depth than the FOT indices. One potential reason for this may be an underlying sensitivity to the number of branches undergoing constriction. The MBW is primarily used as a measure of ventilation heterogeneity (Robinson et al., 2013), meaning parameters such as $s_{\text {cond }}$ should naturally respond more strongly to the magnitude of difference in time constants between different regions than to the relative size of these regions (Otis et al. 1956). Conversely, due to the parallel nature of calculations in the FOT model, the outputs should naturally show sensitivity to the total number of constricted branches. If this is one of the driving mechanistic differences between the two tests, the understanding of it could be quite valuable. However, experimental evidence would be needed to fully test the hypothesis.

\subsection{Behaviour of the Resistance Curve}

Through the simulations, interesting comparative behaviour was seen between the resistance and inertance curves. As seen in Fig. 6 the reactance curve appears to always increase monotonically with frequency, while the resistance curve does not, greatly changing shape under different bronchoconstriction scenarios. Within clinical studies of the FOT the difference between low and high frequency resistance is commonly used as a marker of distal airway constriction. However, the choice of specific markers for low and high varies greatly between studies. Many, such as this study, use $5 \mathrm{~Hz}$ and $20 \mathrm{~Hz}$ (Crim et al. 2011, Kolsum et al., 2009, Shi et al., 2012). However, many other studies use different frequency markers (Bhatawadekar et al., 2015, Delacourt et al., 2001).

Considering the shape of resistance curves, the exact choice of marker appears 
important. This means that indices such as $R_{5}-R_{20}$ can be significantly affected by small changes to the high frequency marker location. This represents an interesting direction for future research, in understanding more precisely how bronchoconstriction alters the shape of the resistance-frequency curve.
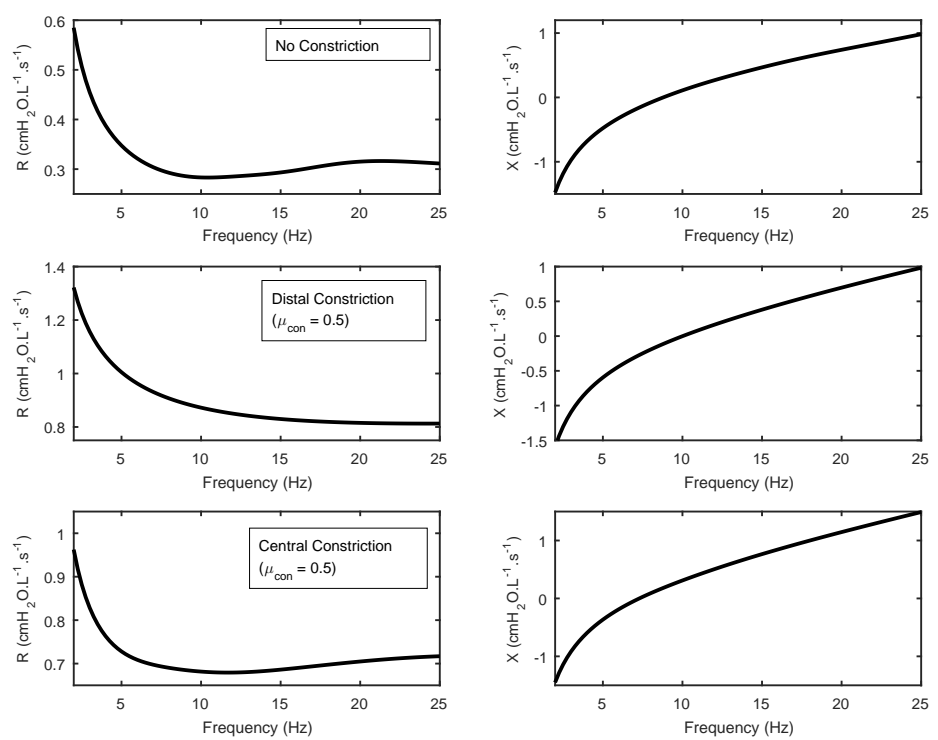

Figure 6: Three different sets of impedance curves: under no constriction (top), distal stochastic constriction, $\mu_{\text {con }}=0.5$ (middle), and central stochastic constriction, $\mu_{\text {con }}=0.5$ (bottom). Consistent monotone behaviour is seen in the reactance curve, while the resistance curve changes in less consistent ways.

\subsection{Limitations of the Study}

When considering the limitations of the models in this study, it should be noted that due to computational constraints the structural lung representation had non-compliant airways. The inclusion of airway compliance would increase total lung resistance, and should lead to slower washout times, increasing the LCI. However, we would not expect $s_{\text {cond }}$ to significantly change, as it is driven by time constant differences, which in our model were most strongly influenced 
by airway constrictions. The MBW model also assumed a static compliance distribution, and simplified behaviour within acinar regions. However, we believe that neither of these assumptions are significant, since the investigation was performed over the tidal breathing range and focused on airway constriction within the conducting zone.

We also believe that the assumption of non-compliant airways did not significantly affect the FOT indices. Original modelling for this study included the effects of airway compliance on FOT indices, using techniques described by Kaczka et al. (2007). However, contributions from airway compliance were seen to be minimal, and thus for simplicity were removed. Further to this, the FOT model did not incorporate contributions to impedance from shunting in the cheeks and upper airways. In clinical applications, cheek shunting effects are minimised by having the patient hold their cheeks throughout the procedure. While useful, this action has been shown to not completely remove potential contributions (Cauberghs and Van de Woestijne, 1983, Oostveen et al., 2003, Peslin et al. 1985). Shunting is known to influence frequency dependence, particularly for resistance measures, due to increased shunting at higher frequencies.

This study primarily focused on bronchoconstriction in the conducting zone, a common occurence in lung diseases such as asthma. However, many other types of lung disease, such as emphysema and COPD, are characterised more strongly by regional compliance heterogeneity, and structural changes within the respiratory zone. The development of models capable of replicating respiratory zone behaviour in sufficient detail to investigate disease at this level is a clear direction for future research.

\subsection{Summary}

In summary, within this study mathematical models of the MBW and FOT were applied to the investigation of bronchoconstriction in the conducting zone of the lungs. In line with previous results in the literature the MBW indices and $R_{5}$ were primarily seen to respond to constriction severity, while $R_{5}-R_{20}$ and the reactance indices were seen to respond strongly to distal airway constriction. 
This suggested the potential utility of joint interpretation of MBW and FOT indices as markers of bronchoconstriction, and generated a hypothesis for future clinical investigations.

\section{Appendix A - MBW indices}

To calculate the major MBW indices, the expired volume, and expired gas concentration must be measured for the duration of the washout phase. The expired gas concentration is typically plotted against time, and referred to as the washout curve. An example washout curve for ${ }^{3} \mathrm{He}$ is given in Fig 7 .

Three major indices are commonly taken from an MBW test: the Lung Clearance Index, $s_{\text {cond }}$ and $s_{\text {acin }}$. The Lung Clearance Index (LCI) is the amount of time taken from the start of the washout phase until mean exhaled concentration over an exhalation drops below 1/40th of the initial exhaled concentration. To normalise between patients, this time is expressed as the number of lung turnovers performed, turnovers being the cumulative expired volume divided by the individual's functional residual capacity (FRC).

The other two measures derived from the MBW are more complex in definition. For each exhalation, a plot of exhaled gas concentration over cumulative expired volume is generated, as seen in Fig 7. Typically this plot undergoes four distinct phases, an initial plateau, a steep rise, a second plateau, and a final rise. An $s_{I I I}$ value is defined as the slope of the third phase of each curve, divided by the mean exhaled concentration over the third phase.

The statistic $s_{\text {cond }}$ is defined as the mean gradient of the $s_{I I I}$ values between turnovers $1.5-6$, and $s_{\text {acin }}$ as the $s_{I I I}$ value from the first exhalation, minus the contribution from the $s_{\text {cond }}$ slope. An example of this is given in Fig 7 We note that typically in the literature, models of the MBW without diffusion produce $s_{I I I}$ curves that extrapolate to zero at turnover 0 (Hamid et al. 2005). As seen in Fig 7 , the $s_{I I I}$ curve from the baseline scenario of this model extrapolates to a small, but non-zero amount. We believe that this is due to the nature of the ventilation model, allowing for variation in ventilation of different acinar 

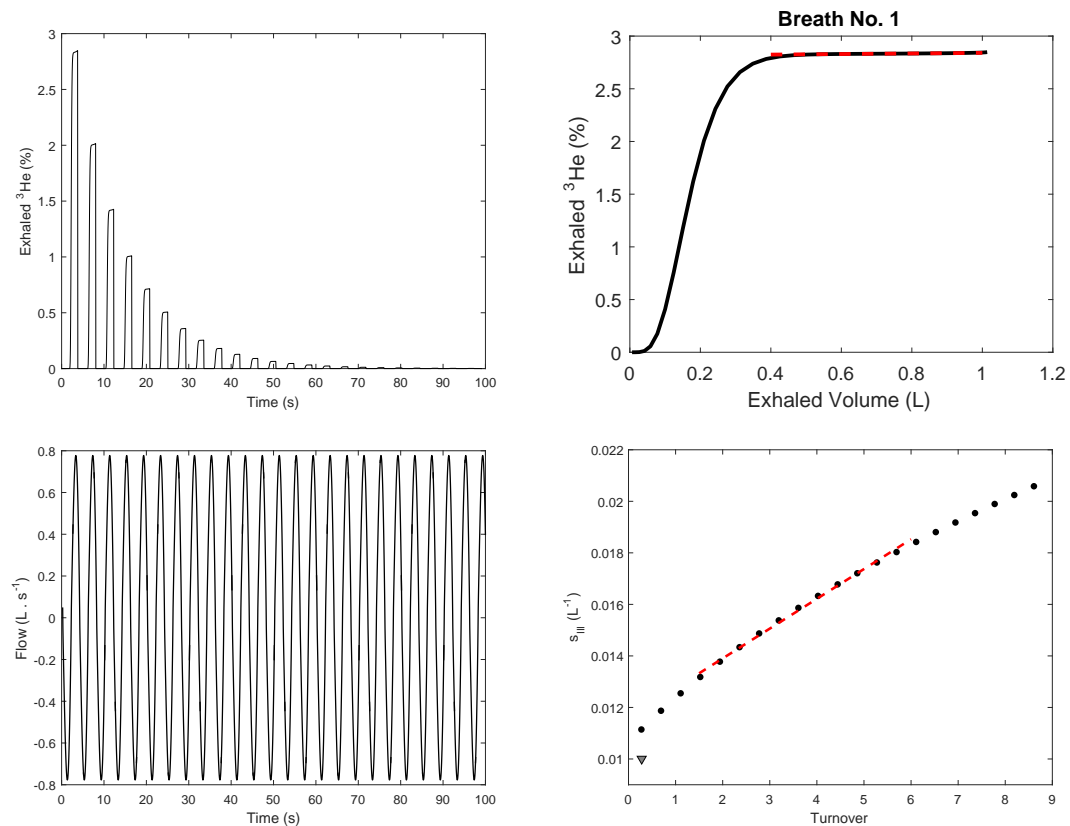

Figure 7: Multiple breath washout indices. A simulated ${ }^{3} \mathrm{He}$ washout under no bronchoconstriction (top left). The exhalation profile for the first breath (right, black line) is given, with the $s_{I I I}$ slope overlaid (red dashed line). Flow rate at the trachea is also given (bottom left). The total set of $s_{I I I}$ values is given (bottom right), with the $s_{\text {cond }}$ slope overlaid (black dashed line), and the index $s_{\text {acin }}$ plotted (grey triangle). This figure was recreated with permission from Foy et al. (2017)

regions, even in the baseline scenario.

\section{Appendix B - Summary Statistics of the Airway Tree}

The structural representation of the lungs used in this study was taken from a healthy subject within the set originally presented by Bordas et al. (2015).

Basic summary statistics of the tree are given in Table 1

\section{Appendix C - Model of the FOT}

Within the FOT model, airway impedance was assumed to result from two major components; oscillatory flow in airway branches, and visco-elastic forces 


\begin{tabular}{ll}
\hline Airway Parameter & Value \\
\hline Lung dimension (medio-lateral axis) & $315 \mathrm{~mm}$ \\
Lung dimension (saggital axis) & $210 \mathrm{~mm}$ \\
Lung dimension (vertical axis) & $279 \mathrm{~mm}$ \\
Maximum branch diameter & $10 \mathrm{~mm}$ \\
Minimum branch diameter & $0.2469 \mathrm{~mm}$ \\
Maximum branch length & $72.95 \mathrm{~mm}$ \\
Minimum branch length & $0.0568 \mathrm{~mm}$ \\
Number of Branches & 78833 \\
Number of Terminal Branches & 39420 \\
Maximum Strahler Order & 12 \\
Maximum Horsfield Order & 26 \\
\hline
\end{tabular}

Table 1: A table of basic statistics of the generated airway tree.

within the acinar regions. Calculations of oscillatory flow in the airway branches were based on approximations from Benade (1968) and Thurston (1952), with the impedance $Z$ in a branch $j$, caused by the propogation of a frequency $f$ calculated as

$$
Z_{j}=\left(i \frac{\omega \rho_{a} l}{\pi r_{j}^{2}}\right)\left(1-F_{v} e^{i \phi r_{j}}\right)^{-1},
$$

where $\rho_{a}$ is the air density, $\omega=2 \pi f, l$ and $r$ are the branch length and radius respectively, and $i=\sqrt{-1}$. The exponential contribution being defined as

$$
F_{v}^{i \phi r}=\frac{2}{r_{v} \sqrt{-i}} \frac{J_{1}\left(r_{v} \sqrt{-i}\right)}{J_{0}\left(r_{v} \sqrt{-i}\right)}, \quad r_{v}=\left(\frac{\omega \rho_{a}}{\mu_{a}}\right)^{\frac{1}{2}} r
$$

where $J_{0}$ and $J_{1}$ are the zeroth and first bessel functions, $\mu_{a}$ is the air viscosity, and $r_{v}$ is the boundary layer thickness.

At the end of each terminal bronchiole, a constant-phase viscoelastic model (Kaczka et al., 2007, Lutchen and Gillis, 1997) was used to account for acinar contributions to impedance such that

$$
Z_{\text {acin }}=\frac{G-i H}{N \omega^{\alpha}}
$$


where $G$ and $H$ are coefficients for tissue damping and elastance respectively, $N$ is the number of terminal bronchioles and

$$
\alpha=\frac{2}{\pi} \tan ^{-1}(H / G) .
$$

For our model, we have taken standard values of $G$ and $H$ from the literature (Kaczka et al., 1997) as 3.75 and $12.5 \mathrm{cmH}_{2}$ O.L ${ }^{-1}$ respectively.

We note that this model does not include effects of gas compression or shunting in the airways. Within initial simulations these effects were included. However, they were seen to have little impact on index values, and led to the introduction of more parameters, and were thus deemed unnecessary.

\section{Acknowledgements}

We wish to thank Dr Rafel Bordas for his valuable insights on the implementation of computational models of the MBW and FOT.

BF was funded by the Rhodes Trust, UK (Rhodes Scholarship, Queensland, 2015). 


\section{References}

Benade, A.H., 1968. On the propagation of sound waves in a cylindrical conduit. J Acoust Soc Am 44, 616-623.

Bhatawadekar, S.A., Leary, D., Maksym, G.N., 2015. Modelling resistance and reactance with heterogeneous airway narrowing in mild to severe asthma. Can J Physiol Pharmacol 93, 207-214.

Bordas, R., Lefevre, C., Veeckmans, B., Pitt-Francis, J., Fetita, C., Brightling, C.E., Kay, D., Siddiqui, S., Burrowes, K.S., 2015. Development and analysis of patient-based complete conducting airways models. PloS ONE 10, e0144105.

Cauberghs, M., Van de Woestijne, K., 1983. Mechanical properties of the upper airway. J Appl Physiol 55, 335-342.

Cavalcanti, J.V., Lopes, A.J., Jansen, J.M., Melo, P.L., 2006. Detection of changes in respiratory mechanics due to increasing degrees of airway obstruction in asthma by the forced oscillation technique. Resp Med 100, 2207-2219.

Crim, C., Celli, B., Edwards, L.D., Wouters, E., Coxson, H.O., Tal-Singer, R., Calverley, P.M., investigators, E., et al., 2011. Respiratory system impedance with impulse oscillometry in healthy and COPD subjects: ECLIPSE baseline results. Resp Med 105, 1069-1078.

Delacourt, C., Lorino, H., Fuhrman, C., Herve-Guillot, M., Reinert, P., Harf, A., Housset, B., 2001. Comparison of the forced oscillation technique and the interrupter technique for assessing airway obstruction and its reversibility in children. Am J Resp Crit Care 164, 965-972.

Fetita, C.I., Prêteux, F., Beigelman-Aubry, C., Grenier, P., 2004. Pulmonary airways: 3-D reconstruction from multislice CT and clinical investigation. IEEE T Med Imaging 23, 1353-1364.

Foy, B.H., Kay, D., Bordas, R., 2017. Modelling responses of the inert-gas washout and mri to bronchoconstriction. Respiratory Physiology \& Neurobiology $235,8-17$. 
Galetke, W., Feier, C., Muth, T., Ruehle, K.H., Borsch-Galetke, E., Randerath, W., 2007. Reference values for dynamic and static pulmonary compliance in men. Resp Med 101, 1783-1789.

Goldman, M.D., Saadeh, C., Ross, D., 2005. Clinical applications of forced oscillation to assess peripheral airway function. Resp Physiol Neurobi 148, 179-194.

Grimby, G., Takishima, T., Graham, W., Macklem, P., Mead, J., 1968. Frequency dependence of flow resistance in patients with obstructive lung disease. J Clin Invest 47, 1455.

Hamid, Q., Shannon, J., Martin, J., 2005. Physiologic basis of respiratory disease. PMPH-USA.

Hopkins, S.R., Henderson, A.C., Levin, D.L., Yamada, K., Arai, T., Buxton, R.B., Prisk, G.K., 2007. Vertical gradients in regional lung density and perfusion in the supine human lung: the slinky effect. J Appl Physiol 103, 240-248.

Horsfield, K., Relea, F.G., Gumming, G., 1976. Diameter, length and branching ratios in the bronchial tree. Resp Physiol 26, 351-356.

Kaczka, D.W., Ingenito, E.P., Israel, E., Lutchen, K.R., 1999. Airway and lung tissue mechanics in asthma: effects of albuterol. Am J Resp Crit Care Med $159,169-178$.

Kaczka, D.W., Ingenito, E.P., Suki, B., Lutchen, K.R., 1997. Partitioning airway and lung tissue resistances in humans: effects of bronchoconstriction. J Appl Physiol 82, 1531-1541.

Kaczka, D.W., Massa, C.B., Simon, B.A., 2007. Reliability of estimating stochastic lung tissue heterogeneity from pulmonary impedance spectra: a forward-inverse modeling study. Ann Biomed Eng 35, 1722-1738.

Kaneko, K., Milic-Emili, J., Dolovich, M., Dawson, A., Bates, D., 1966. Regional distribution of ventilation and perfusion as a function of body position. J Appl Physiol 21, 767-777. 
Kolsum, U., Borrill, Z., Roy, K., Starkey, C., Vestbo, J., Houghton, C., Singh, D., 2009. Impulse oscillometry in COPD: identification of measurements related to airway obstruction, airway conductance and lung volumes. Resp Med 103, 136-143.

Lutchen, K.R., Gillis, H., 1997. Relationship between heterogeneous changes in airway morphometry and lung resistance and elastance. J Appl Physiol 83, $1192-1201$.

Mitchell, J.H., Hoffman, E.A., Tawhai, M.H., 2012. Relating indices of inert gas washout to localised bronchoconstriction. Resp Physiol Neurobi 183, 224-233.

Mittman, C., Edelman, N.H., Norris, A.H., Shock, N.W., 1965. Relationship between chest wall and pulmonary compliance and age. J Appl Physiol 20, $1211-1216$.

Musch, G., Layfield, J.D.H., Harris, R.S., Melo, M.F.V., Winkler, T., Callahan, R.J., Fischman, A.J., Venegas, J.G., 2002. Topographical distribution of pulmonary perfusion and ventilation, assessed by PET in supine and prone humans. J Appl Physiol 93, 1841-1851.

Oostveen, E., MacLeod, D., Lorino, H., Farre, R., Hantos, Z., Desager, K., Marchal, F., et al., 2003. The forced oscillation technique in clinical practice: methodology, recommendations and future developments. Eur Resp J 22, $1026-1041$.

Otis, A.B., McKerrow, C.B., Bartlett, R.A., Mead, J., McIlroy, M., Selverstone, N., Radford, E., 1956. Mechanical factors in distribution of pulmonary ventilation. J Appl Physiol 8, 427-443.

Pedley, T., Schroter, R., Sudlow, M., 1970. The prediction of pressure drop and variation of resistance within the human bronchial airways. Resp Physiol 9, $387-405$.

Peslin, R., Duvivier, C., Gallina, C., Cervantes, P., 1985. Upper airway artifact in respiratory impedance measurements. Am Rev Respir Dis 132, 712-714. 
Robinson, P.D., Latzin, P., Verbanck, S., Hall, G.L., Horsley, A., Gappa, M., Thamrin, C., Arets, H.G., Aurora, P., Fuchs, S.I., et al., 2013. Consensus statement for inert gas washout measurement using multiple-and singlebreath tests. Eur Resp J 41, 507-522.

Shi, Y., Aledia, A.S., Tatavoosian, A.V., Vijayalakshmi, S., Galant, S.P., George, S.C., 2012. Relating small airways to asthma control by using impulse oscillometry in children. J Allergy Clin Immun 129, 671-678.

Smith, H., Reinhold, P., Goldman, M., 2005. Forced oscillation technique and impulse oscillometry. Eur Resp Mon 31, 72.

Strahler, A.N., 1957. Quantitative analysis of watershed geomorphology. Eos T Am Geophys Un 38, 913-920.

Swan, A.J., Clark, A.R., Tawhai, M.H., 2012. A computational model of the topographic distribution of ventilation in healthy human lungs. J Theor Biol $300,222-231$.

Tawhai, M.H., Hunter, P., Tschirren, J., Reinhardt, J., McLennan, G., Hoffman, E.A., 2004. CT-based geometry analysis and finite element models of the human and ovine bronchial tree. J Appl Physiol 97, 2310-2321.

Tawhai, M.H., Hunter, P.J., 2001. Multibreath washout analysis: modelling the influence of conducting airway asymmetry. Resp Physiol 127, 249-258.

Thurston, G.B., 1952. Periodic fluid flow through circular tubes. J Acoust Soc Am 24, 653-656.

Verbanck, S., Paiva, M., 1990. Model simulations of gas mixing and ventilation distribution in the human lung. J Appl Physiol 69, 2269-2279.

Verbanck, S., Schuermans, D., Meysman, M., Paiva, M., Vincken, W., 2004. Noninvasive assessment of airway alterations in smokers: the small airways revisited. American journal of respiratory and critical care medicine 170, $414-419$. 
Verbanck, S., Schuermans, D., Van Muylem, A., Melot, C., Noppen, M., Vincken, W., Paiva, M., 1998. Conductive and acinar lung-zone contributions to ventilation inhomogeneity in COPD. Am J Resp Crit Care Med 157, $1573-1577$.

Verbanck, S., Schuermans, D., Van Muylem, A., Paiva, M., Noppen, M., Vincken, W., 1997. Ventilation distribution during histamine provocation. Journal of Applied Physiology 83, 1907-1916.

\section{Figure Legends}

